\title{
Fiber-modes and fiber-anisotropy characterization using low-coherence interferometry
}

\author{
Y.Z. Ma • Y. Sych • G. Onishchukov • \\ S. Ramachandran · U. Peschel • B. Schmauss • G. Leuchs
}

Received: 27 December 2008 / Revised version: 23 March 2009 / Published online: 7 May 2009

(C) The Author(s) 2009. This article is published with open access at Springerlink.com

\begin{abstract}
An optical low-coherence interferometry technique has been used to simultaneously resolve the mode profile and to measure the intermodal dispersion of guided modes of a few-mode fiber. Measurements are performed using short samples of fiber (about $50 \mathrm{~cm}$ ). There is no need for a complex mode-conversion technique to reach a high interference visibility. Four LP mode groups of the few-mode fiber are resolved. Experimental results and numerical simulations show that the ellipticity of the fiber core leads to a distinct splitting of the degenerate high-order modes in group index. For the first time, to the best of our knowledge, it has been demonstrated that degenerate LP11 modes are much more sensitive to core shape variations than the fundamental modes and that intermodal dispersion of high-
\end{abstract}

\author{
Y.Z. Ma $(\bowtie) \cdot$ U. Peschel · G. Leuchs \\ Institute of Optics, Information and Photonics, University \\ of Erlangen-Nuremberg, Staudtstr. 7/B2, 91058 Erlangen, \\ Germany \\ e-mail: yuzhao.ma@mpl.mpg.de \\ Fax: +49-(0)9131-6877199 \\ G. Onishchukov · U. Peschel · G. Leuchs \\ Max Planck Institute for the Science of Light, \\ Günther-Scharowsky-Str. 1/Bau 24, 91058 Erlangen, Germany
}

Y. Sych · G. Onishchukov · U. Peschel · B. Schmauss · G. Leuchs Erlangen Graduate School in Advanced Optical Technologies (SAOT), Erlangen, Germany

Y. Sych · B. Schmauss

Chair for Microwave Engineering, University

of Erlangen-Nuremberg, Cauerstr. 9, 91058 Erlangen, Germany

S. Ramachandran

Department of Photonics Engineering, Technical University of Denmark, Фrsteds Plads, Building 345v, 2800 Kgs. Lyngby, Denmark order degenerate modes can be used for characterizing the anisotropy of an optical waveguide.

PACS $42.25 . \mathrm{Hz} \cdot 42.81 . \mathrm{Cn} \cdot$ 42.81.Gs

\section{Introduction}

When multiple transverse modes are simultaneously present in an optical fiber, it is important to characterize the dispersion properties of all guided modes. For example, it is well known that in a multimode optical fiber the transverse modes possess different characteristic group velocities. The knowledge of the intermodal dispersion characteristics of multimode fibers is of interest for the design of optical transmission systems, as well as for other applications such as dispersion compensation [1], fiber refractive-index profile characterization [2], and a variety of in-fiber devices [3]. In this context it is necessary to identify the individual transverse modes $[2,4]$ to precisely characterize the dispersion properties.

On the other hand, light beams, possessing special polarization, have recently attracted a great deal of interest. Several fiber techniques have been developed to generate such beams while employing higher-order modes of respective shapes [5-7]. A full characterization of the mode profiles as well as the polarization state of these modes is essential to successfully apply these novel techniques.

Optical low-coherence interferometry has been used to characterize the dispersion properties of an optical fiber since many years. The idea is that the excited modes in a high-order-mode fiber can be separated in time owing to their group-delay difference. By sweeping the wavelength, the group velocity dispersion can be derived. This technique can be classed into two catalogs: time-domain interferometry and frequency-domain interferometry. Here we shortly 


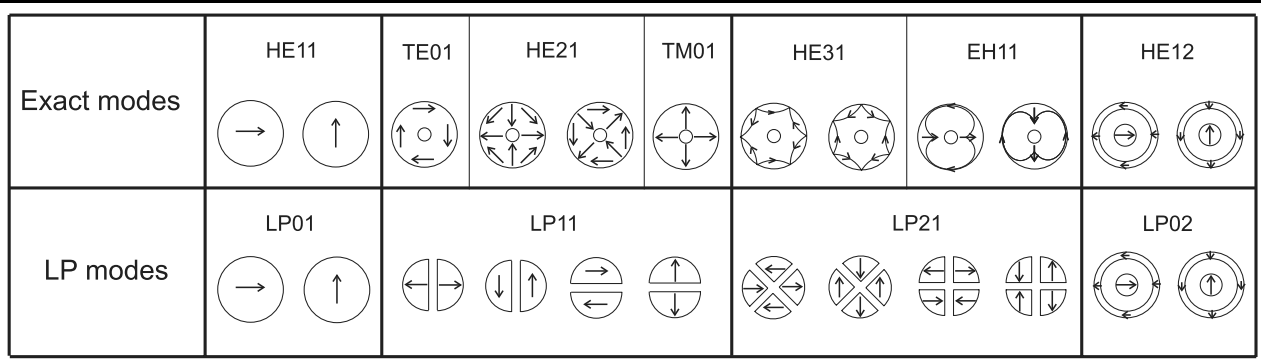

Fig. 1 Modes of a circular step-index fiber. The first row represents exact vector solutions of Maxwell's equations. The second row represents common scalar modes which can be obtained by solving scalar

review these techniques. In time-domain interferometry the optical delay line in the reference arm of the interferometer is introduced to get the interference between the reference beam and the respective mode of the fiber in the other arm. Several variations of this technique have been reported $[2,4,8]$. In this technique either complex detection systems had to be used to distinguish the fiber modes or it had to be assumed that the dispersion properties of the fiber modes were well known according to the prior knowledge of the fiber profile. In traditional frequency-domain interferometry $[9,10]$ the experimental setups were relatively simpler because the interference patterns were observed by an optical spectrum analyzer and no reference arm was required. The theoretic background of the technique is that different frequency components in the light source propagate with different speed inside the fiber, which results in a frequencydependent time delay. However, without the mode identification system, only a limited number of modes, i.e. the polarization mode dispersion of the fundamental mode or the intermodal dispersion of the lowest two LP modes, could be measured. In the new types of frequency-domain interferometry, the Fourier transform [11] and the pulse stretching technique [12] were used to extract the mode information and to obtain a high temporal resolution. However, in these techniques, no accurate mode profiles were obtained. In [13] the frequency-domain technique and the mode detection system were applied simultaneously. However a fiber pigtail had to be used to pick up the intensity distribution of the transverse fiber modes and the spatial accuracy of the mode profile detection was limited.

In the present work we proposed a time-domain lowcoherence interferometry using the traditional phase-shifting method for direct measurement of the group-index difference and of the mode intensity profile of a short piece of special fiber. Furthermore, the polarization distribution of each mode was analyzed. We identified four LP mode groups and analyzed the polarization properties of vector modes in the LP11 mode group. The measurement technique is also feasible for characterization of traditional glass fibers and photonic crystal fibers without any prior knowledge of fiber
Maxwell's equations under weakly-guiding condition. They can be regarded as superpositions of the exact modes shown above

properties. On the other hand it was predicted recently in $[14,15]$ that LP1m modes were more susceptible to asymmetries of the fiber core than the fundamental modes. Using the proposed technique we experimentally demonstrated this effect.

In Sect. 2 we discuss the topology and classification of modes in circular waveguides based on the example of a step-index fiber. In Sect. 3 the measurement principle and the algorithm used for the reconstruction of the mode profiles and of the mode intensities are described in details. In Sect. 4 the measured mode profiles and intermodal dispersion are presented and compared with the results of numerical simulations.

\section{Theoretical background}

\subsection{Step-index fiber}

Step-index fibers have the simplest index profile. Figure 1 shows the first few modes of a circular step-index fiber [1]. The true vectorial eigenmodes are displayed in the first row. The arrows represent the electric field. Each eigenmode has a unique propagation constant except of the even and odd modes of the hybrid modes (HE and $\mathrm{EH}$ ) which have different polarization patterns and are always degenerate in a perfectly circular fiber. The modes shown in the second row are commonly-used linearly-polarized (LP) modes derived by using a scalar approximation [16]. These LP modes are formed by the coherent superpositions of the true vectorial eigenmodes shown above. They represent approximate solutions of Maxwell's equations valid for small core-cladding index steps. In the weakly-guiding regime the propagation constants of the modes in a perfectly circular fiber depend only on the modal intensity pattern and are independent of the polarization.

The normalized frequency $V$ and the normalized propagation constant $b_{\text {neff }}$ are described as

$V=a k\left(n_{\mathrm{co}}^{2}-n_{\mathrm{cl}}^{2}\right)^{\frac{1}{2}}$ 


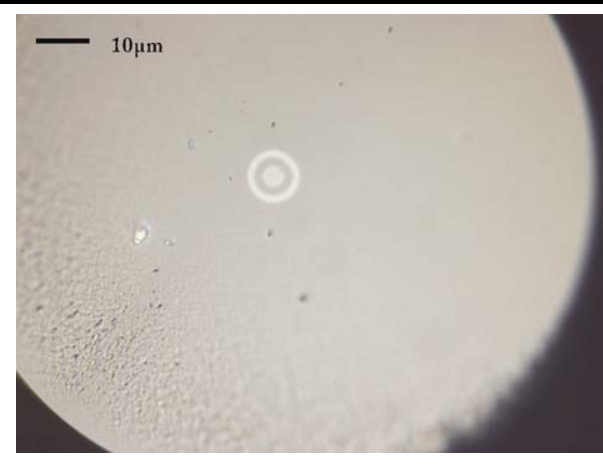

Fig. 2 Cross-section image of the tested few-mode fiber

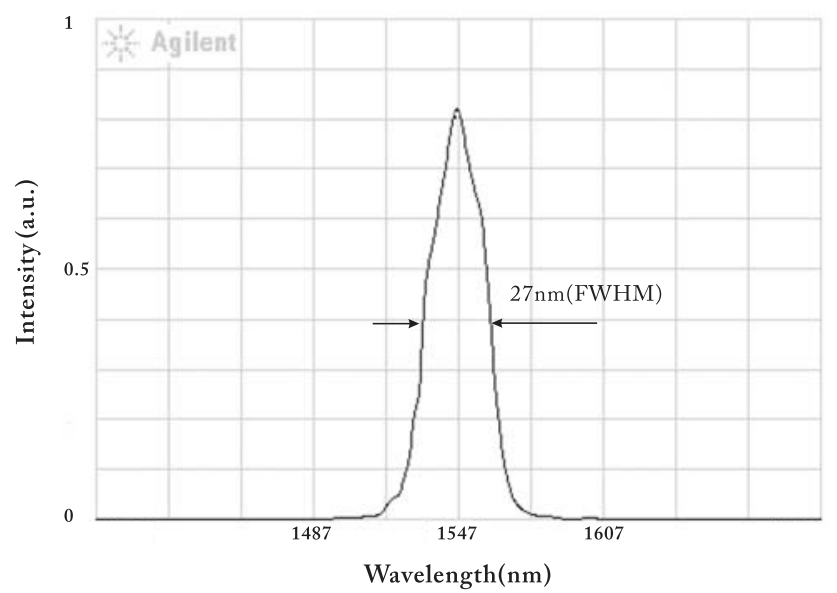

Fig. 3 Spectrum of the low-coherence broadband light source used in the experiments

$$
\begin{aligned}
b_{\text {neff }} & =\left(n_{\text {eff }}^{2}-n_{\mathrm{cl}}^{2}\right) /\left(n_{\mathrm{co}}^{2}-n_{\mathrm{cl}}^{2}\right) \\
& \approx\left(n_{\text {eff }}-n_{\mathrm{cl}}\right) /\left(n_{\mathrm{cl}} \Delta\right)
\end{aligned}
$$

Here $a$ is the core radius; $k=2 \pi / \lambda$ is the wavenumber in vacuum; $n_{\mathrm{co}}$ is the refractive index of the core and $n_{\mathrm{cl}}$ is the refractive index of the cladding. The effective in$\operatorname{dex} n_{\text {eff }}=\beta / k$ is defined by the propagation constant $\beta$. $\Delta=\left(n_{\mathrm{co}}-n_{\mathrm{cl}}\right) / n_{\mathrm{cl}}$ represents the contrast between core and cladding. In weakly-guiding waveguides with $\Delta \ll 1$ the simplification given in (2) can be used. The effective index of all modes is in between the index of the cladding $\left(b_{\text {neff }}=0\right)$ and the index of the core $\left(b_{\text {neff }}=1\right)$, i.e. $n_{\mathrm{cl}} \leq$ $n_{\text {eff }} \leq n_{\text {co }}$.

Another important dispersion parameter is the group index $n_{g}$, defined as

$n_{g}=n_{\mathrm{eff}}-\lambda\left(d n_{\mathrm{eff}} / d \lambda\right)$

It has been shown in $[16,17]$ that the sum of material dispersion and waveguide dispersion gives the total chromatic dispersion of the mode. The material dispersion is the same for all fiber modes, and therefore, it could be neglected when the group-index difference between the modes is to be studied. Without taking into account the material dispersion, i.e. the

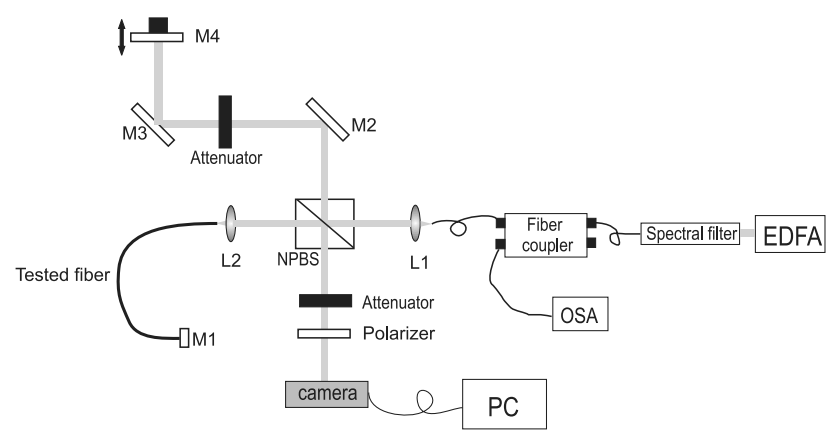

Fig. 4 Low-coherence interferometric setup for intermodal-dispersion measurement with mode profile detection. EDFA-erbium-doped fiber amplifier; NPBS-non-polarizing beam splitter; OSA-optical spectrum analyzer; L1, L2-lenses; M1-gold mirror; M2, M3, M4-dielectric mirrors

frequency dependence of $n_{\mathrm{co}}$ and $n_{\mathrm{cl}}, V$ changes monotonously by $\lambda$ and $d V / d \lambda=-V / \lambda$, and hence,

$$
\begin{aligned}
n_{g} & =n_{\mathrm{eff}}-\lambda\left(d n_{\mathrm{eff}} / d V\right)(d V / d \lambda) \\
& =n_{\mathrm{eff}}+V\left(d n_{\mathrm{eff}} / d V\right)
\end{aligned}
$$

Therefore, the group index on account of waveguide dispersion can be basically deduced from the derivative $d n_{\text {eff }} / d V$. Similar to the normalized propagation constant $b_{\text {neff }}$ we can define the normalized group delay $b_{\text {ng }}$ [16]:

$$
\begin{aligned}
b_{\text {ng }} & =\left(n_{g}^{2}-n_{\mathrm{cl}}^{2}\right) /\left(n_{\mathrm{co}}^{2}-n_{\mathrm{cl}}^{2}\right) \\
& \approx\left(n_{g}-n_{\mathrm{cl}}\right) /\left(n_{\mathrm{cl}} \Delta\right)
\end{aligned}
$$

For a few-mode fiber there is usually a considerable difference between effective indices and group indices.

\subsection{Multimode fiber}

Recently high-order-mode fibers with anomalous dispersion properties [18] have found applications in dispersion compensation, high-power fiber lasers and all-fiber mode converters. All these applications are based on modifying the dispersion properties of specific fiber modes by tailoring the fiber refractive-index profile. In such special fibers, different modes may exhibit significantly different dispersive behavior. In this paper we investigated a fiber with a special index profile: a central core and an outer ring. The cross-section image of this fiber is shown in Fig. 2. This fiber was designed for manufacturing polarization-insensitive fiber gratings [19].

\section{Experimental setup and measurement technique}

In the current work we investigate the intermodal dispersion and the mode profiles of a few-mode optical fiber using lowcoherence interferometry. As a broadband unpolarized light 
Fig. 5 The interferogram corresponding to intensity of one single pixel detected by the InGaAs camera for a LP01 mode (red) and the envelopes reconstructed using (7) (blue)

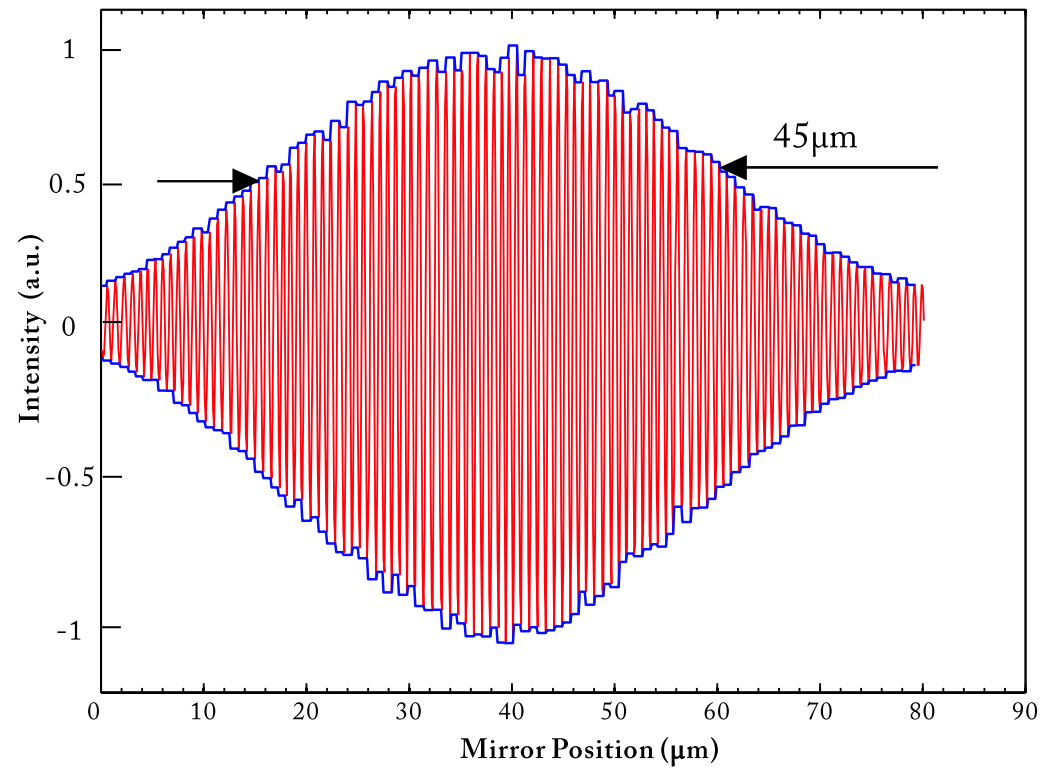

source we used an erbium-doped fiber amplifier (EDFA), the spectrum of which was flattened by a tunable spectral filter. The optimized spectrum of the spontaneous emission of the EDFA, shown in Fig. 3, had a quasi-Gaussian shape with $27-\mathrm{nm}$ bandwidth centered at $1547 \mathrm{~nm}$. A Michelson interferometer was composed of the few-mode fiber in the fiber arm and a free-air path in the reference arm, as shown in Fig. 4. A step-motor translation stage was used to control the path length. In the fiber arm, the light was coupled into the tested fiber and was reflected back at the end of the fiber by a gold layer produced in a sputtering machine. The interference pattern of the beams at the output of the interferometer was recorded by an InGaAs camera. As the variable delay was changed, an interference pattern appeared when the optical path of the reference beam matched that of the fiber beam for a certain transversal mode within the coherence length of the light source. Other modes excited in the fiber produced a background which just decreased the interference contrast. In Fig. 5 the light intensity measured at one camera pixel as a function of delay is shown when matching was achieved for the LP01 mode. Due to the high dynamic range of the InGaAs camera, we were able to resolve all transverse fiber modes using an appropriate offset of the excitation without any additional frequency-filtering electronic circuit to enhance the visibility of the interference fringes [4].

A simple algorithm using the interference maximum and minimum was used to reconstruct the mode profile and to calculate the mode intensity: the intensity of the interference signal of the two arms $I_{\mathrm{nr}}$, the intensity of the $n$th fiber mode $I_{n}$, and the intensity of the reference beam $I_{r}$ follow the relation

$I_{\mathrm{nr}}=I_{0}+I_{r}+I_{n}+2 \sqrt{I_{r} I_{n}} \cos \varphi$ where $I_{0}$ is the intensity of all other fiber modes, the optical paths of which do not match the optical path of the reference arm; the intensity of the reference beam $I_{r}$ was obtained at the beginning of the measurement by blocking the fiber arm; $\varphi$ is the relative phase between the two beams which might depend on transverse position, giving rise to the appearance of fringes in the detected image; $I_{\mathrm{nr}}$ was obtained by the camera in real time. According to the $N$-bucket phase-shifting method [20] we used the following algorithm to find the interference maximum and minimum, and hence, the mode intensity distribution,

$I_{\operatorname{nr}(\max )}=\operatorname{Max}_{i=0}^{N-1}\left(I_{\operatorname{nr}(i)}\right)=I_{0}+I_{r}+I_{n}+2 \sqrt{I_{r} I_{n}}$,

$I_{\mathrm{nr}(\min )}=\operatorname{Min}_{i=0}^{N-1}\left(I_{\mathrm{nr}(i)}\right)=I_{0}+I_{r}+I_{n}-2 \sqrt{I_{r} I_{n}}$,

$M=\left(I_{\mathrm{nr}(\max )}-I_{\mathrm{nr}(\min )}\right) / 2=2 \sqrt{I_{r} I_{n}}$,

$I_{n}=M^{2} / 4 I_{r}$

where $I_{\operatorname{nr}(i)}$ is the intensity of the interference signal for the $i$ th step of the translation stage in the reference arm. The step size was $0.1 \mu \mathrm{m}$ and a 9-bucket algorithm was used to find $I_{\mathrm{nr}(\max )}$ and $I_{\mathrm{nr}(\min )}$. We reconstructed the two-dimensional mode profile by using $I_{\mathrm{nr}(\max )}$ and $I_{\mathrm{nr}(\min )}$ for each single pixel of the images detected by the camera. The blue lines in Fig. 5 are an example of reconstructed envelops of the interference signal. They correspond to the values of $I_{\mathrm{nr}(\max )}$ and $I_{\mathrm{nr}(\mathrm{min})}$ and have a full width at half maximum (FWHM) of $45 \mu \mathrm{m}$. The total power of a certain transverse mode $P_{n}$ is 
Fig. 6 Measured mode

intensity profiles and intermodal dispersion of the few-mode fiber at $1547 \mathrm{~nm}$ using unpolarized beam excitation

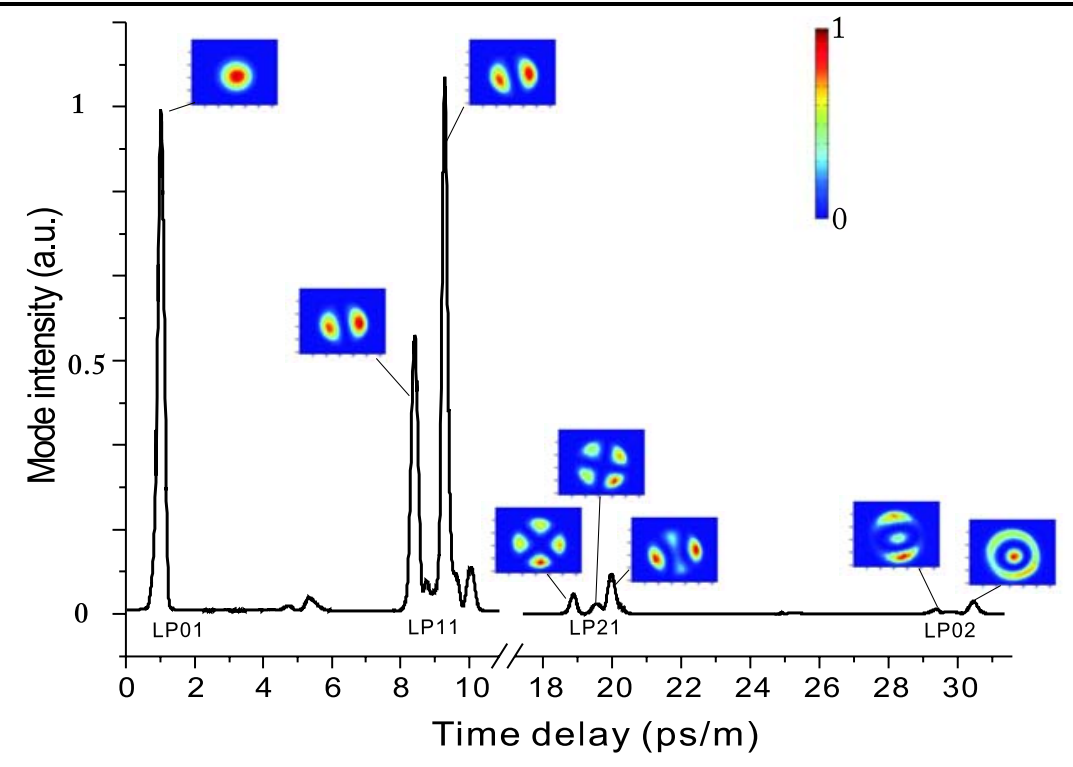

proportional to the sum of the intensities recorded in all the pixels in the transverse plane:

$P_{n}=\sum_{j} \sum_{k} I_{n(j k)}$

where $j$ and $k$ indicate the coordinates of a camera pixel. Thus, it was possible to reconstruct both the mode profile and the mode intensity of the fiber.

Furthermore, because the delay of each peak corresponds to the group delay of the respective mode, we were able to characterize the intermodal dispersion using the relative position of those peaks. The group-index difference between the nth mode and LP01 mode $\Delta n_{g(n)}$ can be described as follows:

$\Delta n_{g(n)}=O P D_{n} / L$

where $O P D_{n}$ is the one-way optical path difference between nth mode and LP01 mode. It is specified by the relative mirror position in the reference arm. $L$ is the length of the fiber.

\section{Results and analysis}

Using the measurement technique described in the Sect. 3, we measured a piece of special fiber with $58 \mathrm{~cm}$ length. The fiber design has been introduced in the Sect. 2.2. In this section we will firstly present some experimental results. Then the results of numerical simulations of modal dispersion and of mode profiles for the tested fiber will be presented. At the end we will discuss the effect of fiber ellipticity on modal dispersion and mode profiles and show that birefringence affects degenerate high-order modes much stronger than the fundamental mode.

\subsection{Mode intensity pattern and group-index difference}

At $1547 \mathrm{~nm}$ four LP mode groups have been identified as illustrated in Fig. 6. In the figure the mode intensity and the mode profile as insets via time delay are shown. The mode intensity profiles are normalized to the maximal intensity in the transverse plane. The measured group indices of LP11, LP21 and LP02 modes relative to the LP01 mode are $\Delta n_{g 11}=0.0025, \Delta n_{g 21}=0.0055, \Delta n_{g 02}=0.0087$. Furthermore, we observe all of the four vector modes of the LP11 mode group separately, although they are almost degenerate in the standard step-index fiber.

In order to investigate the polarization properties of the four modes within the LP11 mode group we adjusted the coupling conditions for excitation of all the four modes with significant magnitude. As shown in Fig. 7(a) they are clearly resolved without using any polarizing component in the setup. To check the polarization properties of the modes, a polarizer was used in front of the camera. The red curve in Fig. 7(b) was obtained by orienting the polarizer along the direction of the dark bar which appeared in the profile of the mode with the largest magnitude. Two modes with the same vertical polarization but with orthogonal intensity patterns, are seen in Fig. 7(b). They correspond to the two HE21 modes. The other two modes, TM01 and TE01, are suppressed. As shown in Fig. 7(b) by the blue curve for the orthogonal polarizer orientation, TM01 and TE01 modes have a polarization orthogonal to that of HE21 modes. They have the same polarization and orthogonal intensity patterns similar to the HE21 mode pair.

It is noted that the TM01, TE01 and the HE21 modes in the LP11 mode group in Fig. 7 appear as typical scalar mode patterns with nearly linear polarization. This can be explained by the anisotropy of the fiber [14, 15], as will be discussed in Sect. 4.3. 

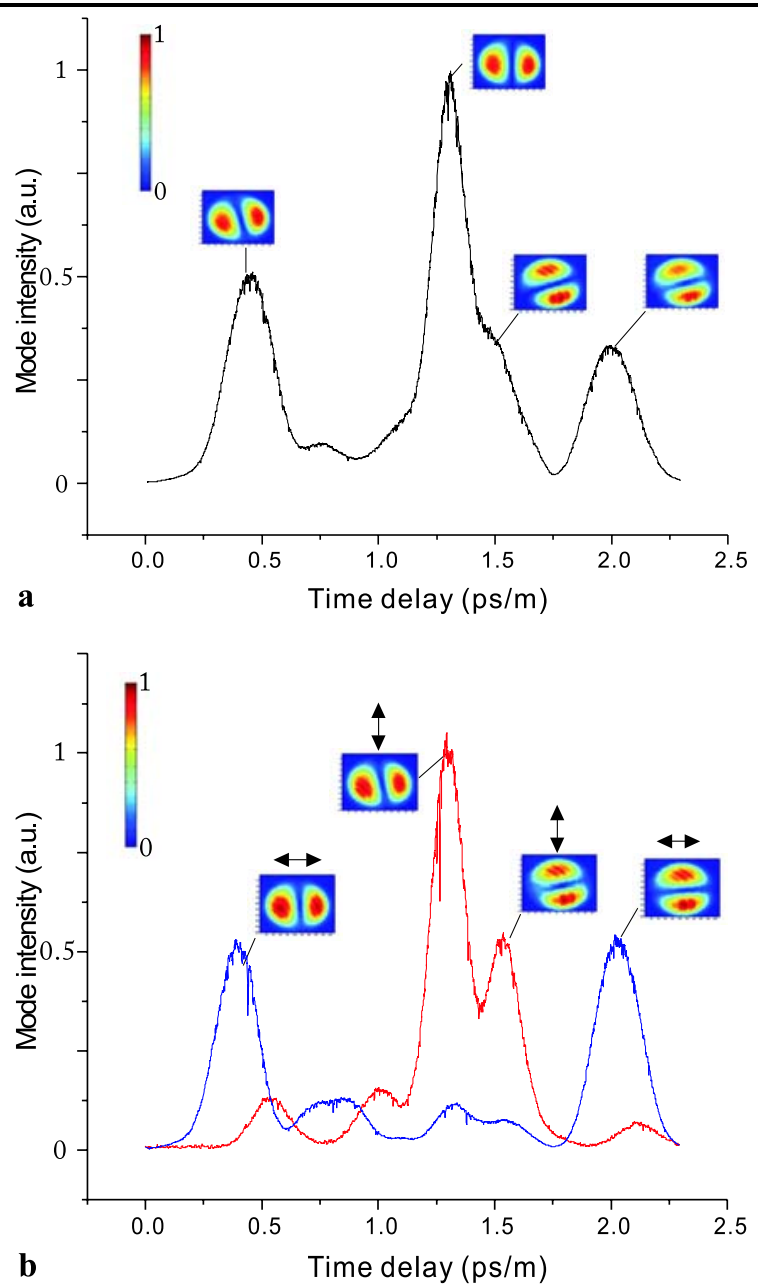

Fig. 7 Intensity profiles and intermodal dispersion for four vector modes within the LP11 mode group using unpolarized beam excitation: (a) no polarizer in front of the camera; (b) with a polarizer in front of the camera. The polarizer axis indicated by the black arrow was oriented vertically or horizontally for red or blue curve, accordingly

\subsection{Modeling of the dispersion properties of the fiber modes}

In this section we will present results of the numerical simulations of the effective indices and group indices of different modes in the tested few-mode fiber. Figure 8 shows the normalized propagation constant $b_{\text {neff }}$ and the normalized group delay $b_{\text {ng }}$ of the lowest 12 modes relative to the normalized frequency $V$ (see (1), (2), (4), (5)). The commercial software "COMSOL Multiphysics" and MATLAB were used for the numerical simulations. The fiber model was created in the graphic environment of COMSOL Multiphysics using the "Eigenfrequency analysis" of the RF application module. The model was then exported as a m-file, which meant the entire modeling session was saved as a sequence of commands. Then the m-file was run in "COMSOL with MATLAB". The codes were modified to study the parameter dependencies. In the calculations $\lambda=1.547 \mu \mathrm{m}$, the core radius $a$, and the refractive index of the cladding $n_{\mathrm{cl}}=1.444$, assumed to be the typical refractive-index value for pure silica at $1.55 \mu \mathrm{m}$ wavelength and room temperature, were kept constant and $V$ was changed by varying the refractive index of the core $n_{\mathrm{co}}$.

The red points in Fig. 8(b) are the experimental data. The vertical line $V=5.94$ corresponds to the designed fiber configuration. In Fig. 8 one can see that the dispersion properties of such a fiber are very different from those of a traditional step-index fiber, especially for the LP11 mode. The splitting of the group indices of the TE01 and HE21 modes, or the HE21 and TM01 modes is about $2 \times 10^{-4}$, while in stepindex fibers the four modes are almost degenerate.

\subsection{Birefringence of high-order modes in an anisotropic fiber}

In this section we will consider the effect of fiber ellipticity on the differential group delay for the HE21 modes. Experimental results presented in Fig. 7 show that the anisotropy of the fiber core results in not only the two-lobe TE01, TM01 and HE21 modes with nearly linear polarization, but also the separation of the HE21even and HE21odd modes to be $260 \mathrm{fs} / \mathrm{m}$ in group delay. This interpretation is confirmed by the mode profiles of the LP21 and LP02 modes displayed in Fig. 6. In practice, both geometric ovality and stress may contribute to this problem. But in order to simplify the model, we assume that the fiber anisotropy is only due to geometric ovality of the fiber core and that the central core and the outer ring core have the same ellipticity. Using the fiber index profile corresponding to $V=5.94$, we studied the dependence of group indices of modes on the ellipticity of the fiber core. The results are shown in Fig. 9(a). The ellipticity is defined as $e=\left(1-b^{2} / a^{2}\right)^{1 / 2}$, where $a$ is the semi-major axis of the elliptical core and $b$ is the semi-minor axis. Our measurements show that the splitting of the HE21even and HE21odd mode in group index is $\Delta n_{\mathrm{gHE} 21}=7 \times 10^{-5}$. Based on this value the estimated ellipticity of the fiber is $e^{2}=0.068$. In Fig. 9 the measured group indices of all resolved modes are shown by red points. As one can see, ellipticity does lead not only to the splitting of the degenerate modes in group index, but also influences the relative group index of each mode group. This is why for $V=5.94$ our experimental data do not fit the calculated group-index value any more, as can be seen in Fig. 9(a). In order to fit the simulation results to our measurement data, we had to slightly change the refractive-index difference between the core and the cladding of the fiber, i.e. $V=6.04$ and calculated the group indices of modes depending on the ellipticity of the fiber core once again (see Fig. 9(b)). As demonstrated in Fig. 9(b), with $V=6.04$ 
Fig. 8 Results of numerical simulations of lowest 12 modes of the tested few-mode fiber: (a) dependence of normalized propagation constant $b_{\text {neff }}$ on normalized frequency $V$;

(b) dependence of the normalized group delay $b_{\text {ng }}$ on normalized cutoff frequency $V$. Vertical line: $V=5.94$; red points: experimental data

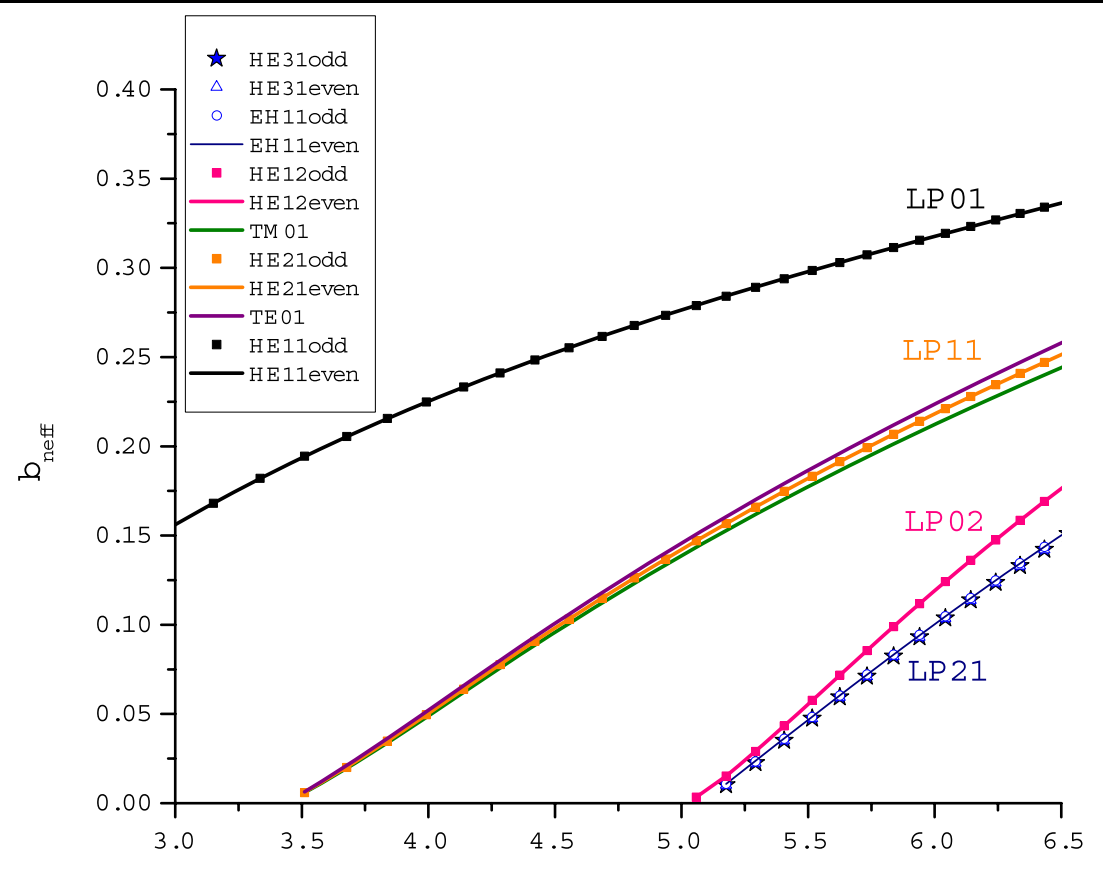

a

$\mathrm{V}$

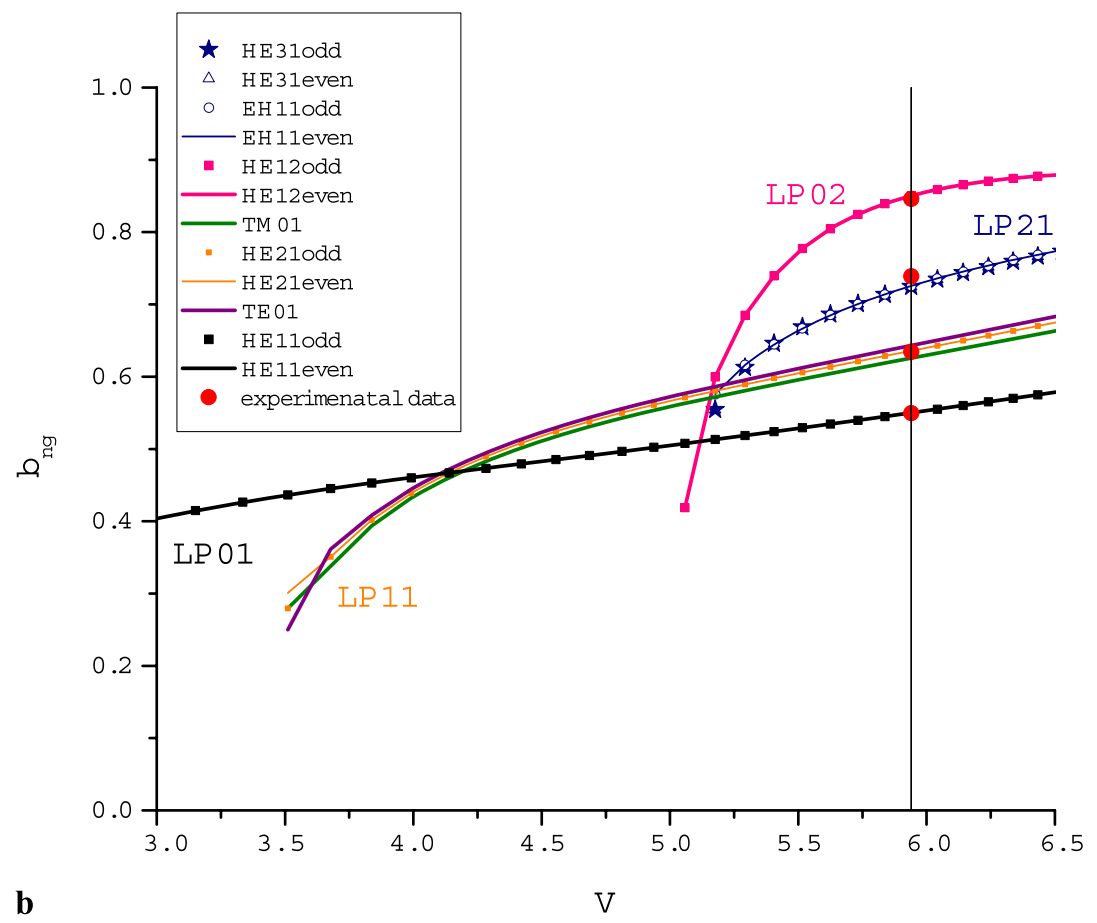

and $e^{2}=0.068$, our measured group indices fit the simulation results very well. Hence we conclude that $V=6.04$ corresponds to the refractive-index profile of the fiber and $e^{2}=0.068$ is the ellipticity inadvertently induced in the manufacturing process.

Our simulations show that the group birefringence of the LP01 mode, $\Delta n_{\mathrm{gLP} 01}$ is just about $7 \times 10^{-6}$. It is ten times smaller than the birefringence of the HE21 modes in the
LP11 mode group, i.e. $\Delta n_{\mathrm{gHE} 21}$ of $7 \times 10^{-5}$. Thus, it is much more efficient to use the value of $\Delta n_{\mathrm{gHE} 21}$ to determine the anisotropy of the fiber because the birefringence effect is about one order of magnitude stronger on HE21 modes than on the fundamental mode. Furthermore, HE21 modes belong to the lowest high-order-mode group and using a Gaussian beam excitation with a slight offset it is possible to excite them with large magnitude. It is also noted 
Fig. 9 Results of numerical simulations on ellipticity dependence of the group index $n_{g}$ for modes of the tested few-mode fiber: (a) for $V=5.94 ;(\mathbf{b})$ for $V=6.04$. Red points: experimental data
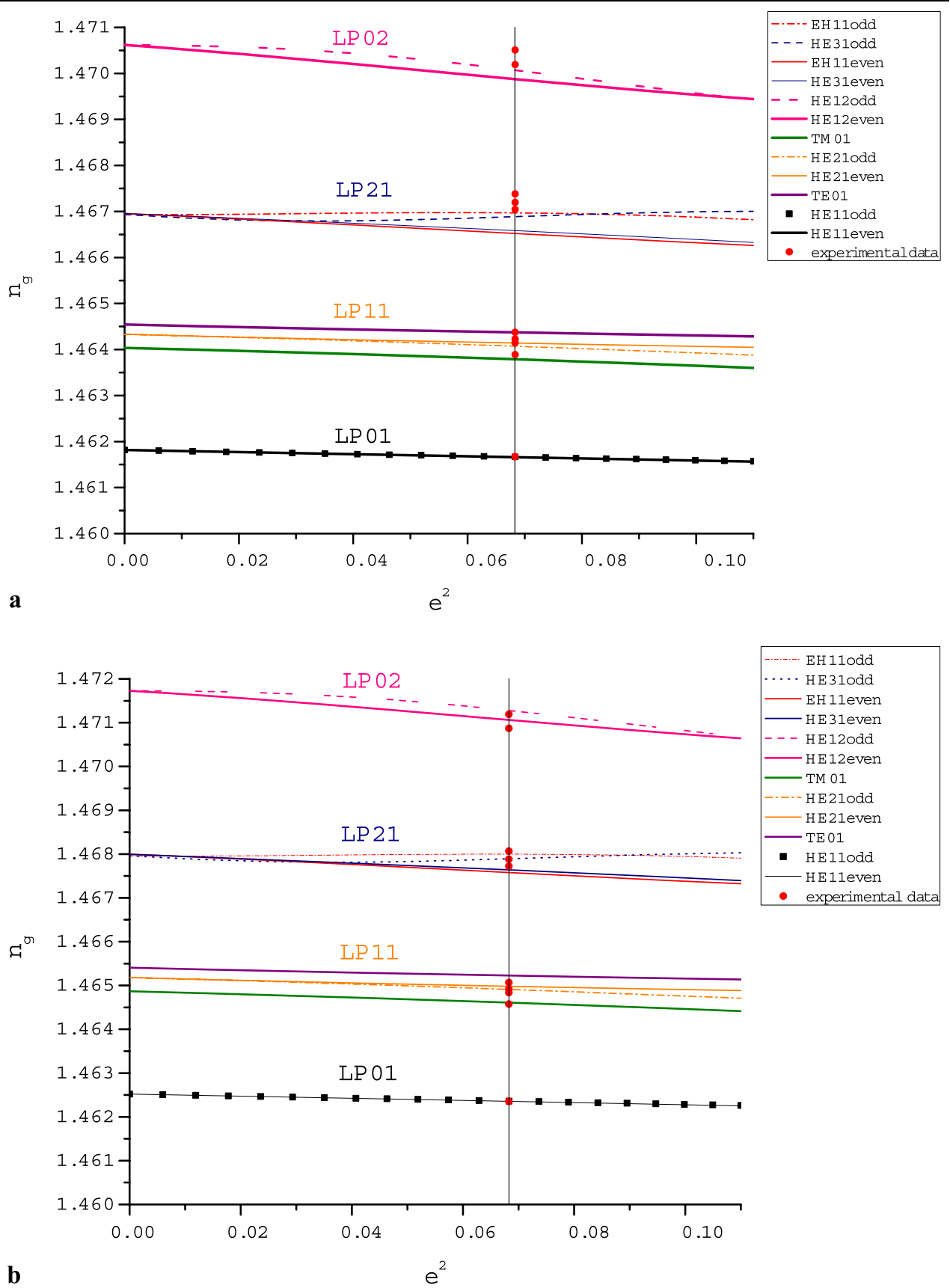

in the simulations that the splitting of HE21 modes in group index being very sensitive to the ellipticity of the fiber, is not that sensitive to the $V$ value of the fiber. This allows us to estimate the anisotropy of a fiber even if the fiber profile is not well known.

In Fig. 10 the calculated mode intensity profiles of the tested fiber with $V=6.04$ and $e^{2}=0.068$ and the measured mode profiles are displayed.

The obtained fiber ellipticity has been confirmed by a separate direct measurement using a frequency-domain interferometer $[9,10]$. A longer piece of fiber (about $8 \mathrm{~m}$ ) has been used to determine the birefringence of the LP01 mode experimentally. The group-index difference of the LP01 mode $\Delta n_{\mathrm{gLP} 01}=10^{-5}$ was obtained. This value is close to $7 \times 10^{-6}$ obtained in the numerical simulations.

\section{Conclusion}

In this paper we have reported on the characterization of fiber modes using low-coherence interferometry. The measured intermodal dispersion has been compared with corresponding simulation results. Very good agreement has 
Fig. 10 Intensity profiles for all guided modes in the tested fiber at $1547 \mathrm{~nm}$ : (a) calculated mode profiles of the fiber with

$V=6.04, e^{2}=0.068$;

(b) measured mode profiles.

Each row shows one mode group; each column shows vector modes in one mode group. White arrows indicate the dominant polarization of the modes

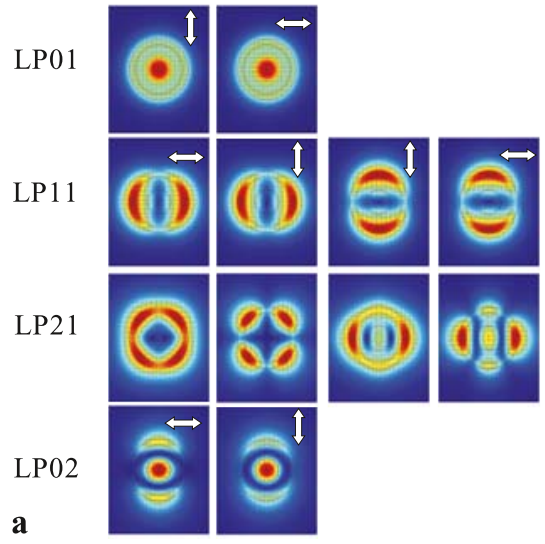

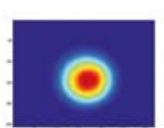
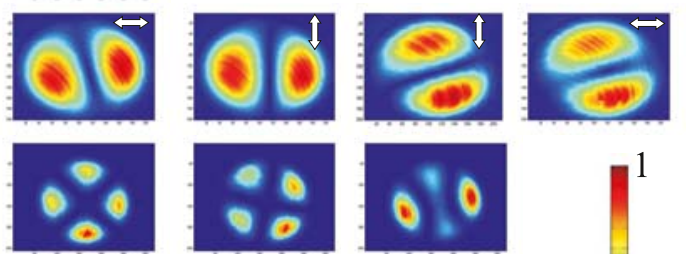

,
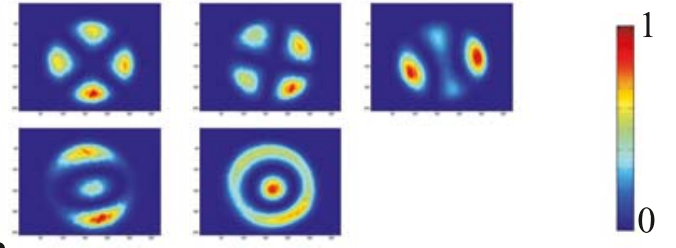

been obtained. The mode profiles and the intermodal dispersion of the degenerate high-order modes offer us information on anisotropy of the fiber which was introduced in the manufacturing process. For the first time, to the best of our knowledge, we demonstrated that the dispersion properties of high-order modes instead of fundamental mode in an optical fiber provide valuable information for the characterization of fiber anisotropy. This makes the work easier, especially when the fiber is short and the birefringence of fundamental modes is too weak to measure.

Open Access This article is distributed under the terms of the Creative Commons Attribution Noncommercial License which permits any noncommercial use, distribution, and reproduction in any medium, provided the original author(s) and source are credited.

\section{References}

1. C.D. Poole, J.M. Wiesenfeld, D.J. DiGiovanni, A.M. Vengsarkar, Optical fiber-based dispersion compensation using higher order modes near cutoff. J. Lightw. Technol. 12, 1746-1758 (1994)

2. Y. Painchaud, P. LeBel, M.A. Duguay, R.J. Black, Time-resolved identification of modes and measurement of intermodal dispersion in optical fibers. Appl. Opt. 31, 2005-2010 (1992)

3. S. Ramachandran, Dispersion-tailored few-mode fibers: A versatile platform for in-fiber photonic devices. J. Lightw. Technol. 23, 3426-3443 (2005)

4. Y. Painchaud, M.A. Duguay, F. Ouellette, Interferometric time measurements of intermodal dispersion in optical fibers by using a CCD photodetector array. Opt. Lett. 17, 1423-1425 (1992)

5. G. Volpe, D. Petrov, Generation of cylindrical vector beams with few-mode fibers exited by Laguerre-Gaussian beams. Opt. Commun. 237, 89-95 (2004)

6. T. Grosjean, A. Sabac, D. Courjon, A versatile and stable device allowing the efficient generation of beams with radial, azimuthal or hybrid polarizations. Opt. Commun. 252, 12-21 (2005)
7. A. Witkowska, S.G. Leon-Saval, A. Pham, T.A. Birks, All-fiber LP $_{11}$ mode convertors. Opt. Lett. 33, 306-308 (2008)

8. P. Hamel, Y. Jaouën, R. Gabet, S. Ramachandran, Optical lowcoherence reflectometry for complete chromatic dispersion characterization of few-mode fibers. Opt. Lett. 32, 1029-1031 (2007)

9. X.D. Cao, D.D. Meyerhofer, Frequency-domain interferometer for measurements of the polarization mode dispersion in single-mode optical fibers. Opt. Lett. 19, 1837-1839 (1994)

10. J.M. Dudley, S.G. Murdoch, Intermodal dispersion and polarization mode dispersion measurements in optical fibers using a self-modelocked Ti:sapphire laser. Opt. Fiber Technol. 2, 80-84 (1996)

11. C. Dorrer, S. Ramachandran, Self-referencing dispersion characterization of multimode structures using direct instantaneous frequency measurement. IEEE Photonics Technol. Lett. 16, 1700$1702(2004)$

12. T.-J. Ahn, Y. Park, D.J. Moss, S. Ramachandran, J. Azaña, Frequency-domain modal delay measurement for higher-order mode fiber based on stretched pulse interference. Opt. Lett. 33, 19-21 (2008)

13. J.W. Nicholson, A.D. Yablon, S. Ramachandran, S. Ghalmi, Spatially and spectrally resolved imaging of modal content in largemode-area fibers. Opt. Express 16, 7233-7243 (2008)

14. S. Golowich, S. Ramachandran, Impact of fiber design on polarization dependence in microbend gratings. Opt. Express 13, 68706877 (2005)

15. A.W. Snyder, J.D. Love, Optical Waveguide Theory (Chapman \& Hall, London, 1983)

16. D. Gloge, Weakly guiding fibers. Appl. Opt. 10, 2252-2258 (1971)

17. D. Gloge, Dispersion in weakly guiding fibers. Appl. Opt. 10, 2442-2445 (1971)

18. S. Ramachandran, S. Ghalmi, J.W. Nicholson, M.F. Yan, P. Wisk, E. Monberg, F.V. Dimarcello, Anomalous dispersion in a solid, silica-based fiber. Opt. Lett. 31, 2532-2534 (2006)

19. S. Ramachandran, S. Golowich, M.F. Yan, E. Monberg, F.V. Dimarcello, J. Fleming, S. Ghalmi, P. Wisk, Lifting polarization degeneracy of modes by fiber design: a platform for polarizationinsensitive microbend fiber gratings. Opt. Lett. 30, 2864-2866 (2005)

20. G. Lai, T. Yatagai, Generalized phase-shifting interferometry. J. Opt. Soc. Am. A 8, 822-827 (1991) 